\title{
BIODEGRADAÇÃO DE GLIFOSATO EM DOIS SOLOS BRASILEIROS
}

\author{
ADEMIR SÉRGIO FERREIRA DE ARAÚJO ** \\ REGINA TERESA ROSIM MONTEIRO ** \\ ROSÂNGELA BLOTA ABAKERLI *** \\ LOURDES SILVESTRE DE SOUZA ****
}

\begin{abstract}
Avaliou-se a biodegradação de Glifosato em amostras de dois solos brasileiros, ambos com e sem histórico de uso prévio do herbicida. Aplicou-se o Glifosato em $75 \mathrm{~g}$ de cada amostra de solo (três repetições) na dosagem recomendada para condição de campo (2,16 kg i.a./ha). A biodegradação foi avaliada monitorando-se a liberação do $\mathrm{CO}_{2}$ pelos microrganismos no período de 32 dias. Durante esse período foram quantificados os resíduos de Glifosato e seu principal metabólito por meio de extração, seguida de análise por Cromatografia a Líquido de Alta Eficiência. Os resultados mostraram que o Glifosato foi degradado pelos microrganismos do solo, com formação de seu metabólito ácido aminometilfosfônico (AMPA). A degradação mostrouse ligeiramente superior em Argissolo que em Latossolo.
\end{abstract}

PALAVRAS-CHAVE: HERBICIDAS-BIODEGRADAÇÃO; CLAE; GLIFOSATO; SOLOS.

* Engenheiro Agrônomo, MsC, Seção de Ecologia, Centro de Energia Nuclear na Agricultura (CENA), Universidade de São Paulo (USP), Piracicaba, SP, Brasil (e-mail: asfaruaj@carpa.ciagri.usp.br)

** Bióloga, PhD, Seção de Ecologia, CENA, USP, Piracicaba, SP, Brasil.

*** Química, PhD, Embrapa - Meio Ambiente, Jaguariúna, SP, Brasil.

**** Técnica de Laboratório, Embrapa - Meio Ambiente, Jaguariúna, SP, Brasil. 


\section{INTRODUÇÃO}

Dentre os processos biológicos que determinam a persistência dos herbicidas no solo, a degradação microbiana constitui o de maior importância. Entretanto, a complexa interação entre microrganismos, substratos e os constituintes do solo influenciam e determinam a magnitude da biodegradação dessas moléculas (SOUZA et al., 1999).

O Glifosato, herbicida pós-emergente, é muito utilizado na agricultura e em áreas não-cultivadas, visando o controle de ervas daninhas anuais e perenes (RUEPPEL et al., 1977). Segundo LIU et al. (1991), a principal via de biodegradação do Glifosato envolve a clivagem da molécula, produzindo o ácido aminometilfosfônico (AMPA) e em seguida água, dióxido de carbono e fosfato (FORLANI et al., 1999).

SOUZA et al. (1999) estudaram a biodegradação de Glifosato e de Imazapir em dois solos com diferentes texturas e submetidos a diferentes doses dos herbicidas. Observaram que a respiração microbiana aumentou na presença dos herbicidas e afirmaram que a microbiota foi capaz de utilizar o Glifosato e o Imazapir como fontes de carbono para seu crescimento.

O Glifosato apresenta persistência variável no ambiente, embora seja degradado pelos microrganismos (NEWTON et al., 1994). Os fatores que influenciam a persistência do Glifosato estão relacionados com sua adsorção ao solo e disponibilidade para biodegradação (SOUZA et al., 1999). Em estudo sobre biodegradação de Glifosato (fase adsorvida e fase não-adsorvida), EBERBACH (1998) observou que houve rápida degradação no primeiro dia, seguida de diminuição até o quadragésimo dia. Segundo o referido autor, a influência da adsorção restringiu a disponibilidade do Glifosato para biodegradação ao longo do tempo. Assim, a meia-vida da molécula depende das fases, sendo a da parte não-adsorvida de 6 a 9 dias e da parte adsorvida de 222 a 835 dias. Os herbicidas quando aplicados repetidamente no solo por vários anos podem ter sua taxa de degradação aumentada em relação aos solos sem a aplicação do produto, pois os microrganismos presentes podem estar mais adaptados à presença do composto e apresentar enzimas específicas para metabolizá-lo. ROBERTSON e ALEXANDER (1994) conduziram estudo para avaliar a ocorrência de degradação acelerada de alguns herbicidas e observaram que o Glifosato foi rapidamente mineralizado após a segunda aplicação no solo.

O objetivo deste trabalho foi avaliar a biodegradação de Glifosato em amostras de dois solos brasileiros, com e sem histórico de aplicação prévia do herbicida em campo.

\section{MATERIAL E MÉTODOS}

\subsection{SOLOS}

Amostras de Argissolo Vermelho-Amarelo, textura média, foram coletadas em área cultivada com pêssego, na Fazenda Experimental da Escola Superior de Agricultura Luiz de Queiroz (ESALQ/ USP), situada no município de Piracicaba/SP. Retiraram-se amostras de subarea com histórico de seis anos de aplicações anuais de Glifosato (ArV6) e outra sem histórico de aplicação (ArV0). Amostras de Latossolo Vermelho, textura argilosa, da Fazenda Experimental do Instituto Agronômico do Paraná (IAPAR), foram coletadas no município de Londrina/PR. Uma amostra foi retirada de subarea sem histórico de aplicação (LV0), cultivada com banana, e outra com histórico de 11 anos de aplicações anuais de Glifosato (LV11), cultivado com soja no sistema de plantio direto. A composição físicoquímica das amostras de solos utilizadas no experimento constam da Tabela 1. 
TABELA 1 - CARACTERÍSTICAS FÍSICAS E QUÍMICAS DAS AMOSTRAS DOS SOLOS ARGISSOLO VERMELHO-AMARELO (ARV) E LATOSSOLO VERMELHO (LV)

\begin{tabular}{|c|c|c|c|c|c|c|c|c|}
\hline \multirow[t]{2}{*}{ Solos } & \multirow{2}{*}{$\begin{array}{c}\text { Aplica a } 0 \\
\text { de Glifosato } \\
\text { (anos) }\end{array}$} & \multicolumn{7}{|c|}{ Caracter sticas } \\
\hline & & $\begin{array}{c}\text { Areia } \\
(\%)\end{array}$ & $\begin{array}{l}\text { Silte } \\
(\%)\end{array}$ & $\begin{array}{c}\text { Argila } \\
(\%)\end{array}$ & $\mathrm{pH}$ & Org & $\begin{array}{l}\text { MatØria } \\
\text { nica }(\mathrm{g} / \mathrm{kg})\end{array}$ & $\begin{array}{c}\text { CTC }^{*} \\
(\mathrm{Mmolc} / \mathrm{kg})\end{array}$ \\
\hline ArV0 & 0 & 41 & 13 & 46 & 5,7 & & 23 & 88 \\
\hline ArV6 & 6 & 49 & 9 & 42 & 5,9 & & 23 & 92 \\
\hline LVO & 0 & 03 & 11 & 86 & 5,6 & & 26 & 118 \\
\hline LV11 & 11 & 01 & 08 & 91 & 5,2 & & 20 & 92 \\
\hline
\end{tabular}

* CTC = Capacidade de troca catiônica.

Em cada subarea foram coletadas, com auxílio de uma pá, subamostras de solo (inteiramente ao acaso) em 15 pontos na profundidade de $0-10 \mathrm{~cm}$ do perfil, formando amostras compostas. As amostras de solo foram peneiradas em malhas de $2 \mathrm{~mm}$ com posterior determinação da umidade presente e da capacidade de campo (COSTA, 1983). As amostras foram acondicionadas em sacos plásticos, fechados frouxamente com barbante e papel e mantidos a temperatura de $4^{\circ} \mathrm{C}$ ( 1 semana) para análises posteriores.

\subsection{BIODEGRADAÇÃO DE GLIFOSATO NO SOLO}

Os experimentos foram conduzidos no Laboratório de Ecotoxicologia, do Centro de Energia Nuclear na Agricultura (CENA/USP). Usou-se a metodologia de quantificação do dióxido de carbono desprendido pela respiração microbiana do solo (BARTHA e PRAMER, 1965). O Glifosato (99\% de pureza) foi aplicado na dosagem de $2,16 \mathrm{mg} \cdot \mathrm{kg}^{-1}$ de solo e distribuído, uniformemente, em sacos plásticos. Foram utilizados $75 \mathrm{~g}$ (peso seco) de cada amostra de solo, colocadas em frascos Erlenmeyer

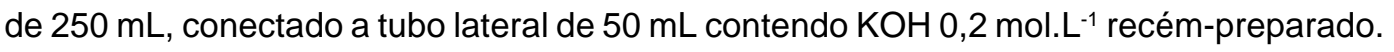

Os frascos Erlenmeyer foram incubados a temperatura de $25^{\circ} \mathrm{C}\left( \pm 2^{\circ} \mathrm{C}\right)$, no escuro e em sala climatizada. O dióxido de carbono liberado e coletado no álcali $(\mathrm{KOH})$ foi amostrado aos 2, 4, 8 16, 24 e 32 dias após o início da incubação do solo, trocando-se os $10 \mathrm{~mL}$ de $\mathrm{KOH}$ contido no tubo lateral do frasco Erlenmeyer por solução recém-preparada. $\mathrm{Os} 10 \mathrm{~mL}$ de $\mathrm{KOH}$ retirados foram colocados em frascos Erlenmeyer de $125 \mathrm{~mL}$, contendo $1,0 \mathrm{~mL}$ de $\mathrm{BaCl}_{2} 1 \mathrm{~mol}^{-\mathrm{L}^{-1}}$ para precipitar o $\mathrm{CO}_{2}$ e 3 gotas de solução de fenolftaleína como indicador. A solução foi titulada com $\mathrm{HCl} 0,1 \mathrm{~mol} \cdot \mathrm{L}^{-1}$ até a viragem, anotando-se o volume gasto. O mesmo procedimento descrito acima foi utilizado para os frascos sem adição de solo (branco). A quantificação do $\mathrm{CO}_{2}$ desprendido foi calculada pela seguinte fórmula (IBAMA, 1990):

$$
\mu \mathrm{g} \mathrm{C}-\mathrm{CO}_{2}=\left[\left(\mathrm{mL} \mathrm{HCl} \mathrm{gasto}_{\text {branco }}\right)-\left(\mathrm{mL} \mathrm{HCl} \text { gasto }_{\text {tratamento }}\right)\right] \times \mathrm{M}_{\mathrm{HCl}} \times 22
$$

\subsection{QUANTIFICAÇÃO DO GLIFOSATO E METABÓLITO}

Para análise de resíduos de Glifosato e seu metabólito ácido aminometil fosfônico (AMPA) foram utilizadas amostras dos mesmos solos, incubadas nas mesmas condições, em quatro frascos Erlenmeyer adicionais para cada solo. As análises foram efetuadas aos $0,8,16$ e 32 dias após a 
aplicação do herbicida. Amostras de $10 \mathrm{~g}$ de solo, em três repetições, foram colocadas em tubos de centrífuga de $75 \mathrm{~mL}$, juntamente com solução de $\mathrm{NH}_{4} \mathrm{OH} 0,25$ mol.L-1 $+\mathrm{KH}_{2} \mathrm{PO}_{4}$ 0,1 mol.L-1 (40 mL). Em seguida, foram agitadas por 90 minutos em agitador horizontal (120 rpm) e centrifugadas a $2000 \mathrm{rpm}$, por 20 minutos. O sobrenadante foi transferido para outro tubo de centrífuga, o pH ajustado para 2,0 com HCl 5 mol.L-1 e centrifugado novamente a $2500 \mathrm{rpm}$ por 10 minutos. Filtrou-se 0 sobrenadante em papel filtro GF/A e o filtrado foi transferido para balão volumétrico de fundo redondo de $300 \mathrm{~mL}$. Repetiu-se o procedimento descrito acima com o resíduo sólido restante no tubo de centrífuga inicial. O volume do filtrado combinado das duas extrações foi reduzido para, aproximadamente, $5 \mathrm{~mL}$ em concentrador rotatório a vácuo a temperatura de $60^{\circ} \mathrm{C}$. O extrato de solo foi transferido para tubo de centrífuga e o pH ajustado entre 1,8 e 2,0 com NH${ }_{4} \mathrm{OH}$ concentrado, sendo centrifugado novamente a 4000 rpm por 10 minutos.

O extrato do solo foi transferido para coluna contendo resina de troca catiônica (Dowex 50W$\mathrm{X8}$ ), com taxa de fluxo regulada de 2,5 $\mathrm{mL} \cdot \mathrm{min}^{-1}$. Em seguida, foram feitas lavagens seqüenciais com porções de $5 \mathrm{~mL}$ de $\mathrm{HCl} 0,01 \mathrm{~mol}^{-\mathrm{L}^{-1}}$ até volume total de $20 \mathrm{~mL}$, descartando-se o eluído. Glifosato e AMPA foram eluídos da coluna, usando-se $\mathrm{HCl} 0,1 \mathrm{~mol}^{-\mathrm{L}^{-1}}(5 \times 20 \mathrm{~mL})$ e coletados em balão de 500 $\mathrm{mL}$. O extrato limpo foi evaporado até secura em evaporador rotatório a vácuo. O resíduo foi redissolvido com $5 \mathrm{~mL}$ de fase móvel $\left(\mathrm{KH}_{2} \mathrm{PO}_{4}\right.$ 0,005 mol.L-1, $4 \%$ metanol, $\mathrm{pH}$ 2,1, ajustado com ácido fosfórico) e colocado em balão volumétrico de $5 \mathrm{~mL}$. $O$ extrato final foi filtrado em seringa provida de membrana Millipore de $0,45 \mu \mathrm{m}$ e armazenado em frascos de vidro de $5 \mathrm{~mL}\left(-20^{\circ} \mathrm{C}\right)$. As amostras foram enviadas para análise de Glifosato e AMPA por Cromatografia a Líquido de Alta Eficiência (COWELL et al., 1986) ao Laboratório de Resíduos de Pesticidas da EMBRAPA-Meio Ambiente.

As análises foram efetuadas em equipamento Shimadzu (modelo LC 10AD), provido de coluna analítica de troca aniônica de sulfonato de estireno divinil-benzeno $(5 \mu \mathrm{m}, 300 \mathrm{~mm} \times 4,6 \mathrm{~mm}$ de diâmetro interno), em temperatura constante de $50^{\circ} \mathrm{C}$. O fluxo da fase móvel foi mantido a $0,5 \mathrm{~mL} \cdot \mathrm{min}^{-1}$ e o volume de amostra injetada foi de $100 \mu \mathrm{L}$.

A determinação de Glifosato e AMPA envolveu, primeiramente, a oxidação da amina primária da estrutura molecular de Glifosato em reator pós-coluna a $38^{\circ} \mathrm{C}$, seguida de derivatização com o-ftalaldeído e 2-mercaptoetanol (OPA-MERC) em espiral de aço inoxidável (2 $\mathrm{m} \times 0,2 \mathrm{~mm}$ de diâmetro interno). Duas bombas foram usadas para obter fluxos de $0,3 \mathrm{~mL} \cdot \mathrm{min}^{-1}$ para o reagente oxidante e o OPAMERC. Usou-se detector de fluorescência, com comprimento de onda de excitação de $350 \mathrm{~nm}$ e emissão de $440 \mathrm{~nm}$, e integrador. Os tempos de retenção para Glifosato e AMPA foram 22 e 44 minutos, respectivamente.

\subsection{DELINEAMENTO EXPERIMENTAL}

O delineamento experimental foi inteiramente casualizado com oito tratamentos e três repetições como segue: 1 - amostra ArV0, com aplicação de Glifosato no laboratório; 2 - amostra ArV0, sem aplicação de Glifosato no laboratório (controle); 3 - amostra ArV6, com aplicação de Glifosato no laboratório; 4 - amostra ArV6, sem aplicação de Glifosato no laboratório (controle); 5 - amostra LV0, com aplicação de Glifosato no laboratório; 6 - amostra LV0, sem aplicação de Glifosato no laboratório (controle); 7 - amostra LV11, com aplicação de Glifosato no laboratório; 8 - amostra LV11, sem aplicação de Glifosato no laboratório (controle). Para comparação de médias foi utilizado o teste de Tukey, ao nível de 5\%, de acordo com PIMENTEL-GOMES e GARCIA (2002).

\section{RESULTADOS E DISCUSSÃO}

Os resultados da biodegradação de Glifosato, obtidos por meio do desprendimento de $\mathrm{CO}_{2}$ (no período de 32 dias) são apresentados na Figura 1a e 1b. Observou-se que, nas amostras dos solos em 
que foi aplicado Glifosato no laboratório a quantidade de $\mathrm{CO}_{2}$ aos 32 dias foi de 0,49 e 0,42 $\mu \mathrm{g} \cdot \mathrm{g}^{-1}$ para os solos ArV6 e ArV0, e de 0,49 e 0,46 $\mu \mathrm{g} \cdot \mathrm{g}^{-1}$ para os solos LV11 e LV0, respectivamente. Nas amostras dos solos em que não foi aplicado Glifosato no laboratório (controles), a quantidade de $\mathrm{CO}_{2}$ aos 32 dias foi de 0,39 e $0,32 \mu \mathrm{g} \cdot \mathrm{g}^{-1}$ para os solos ArV6 e ArV0, e de 0,40 e 0,38 $\mu \mathrm{g} \cdot \mathrm{g}^{-1}$ para os solos LV11 e LV0, respectivamente. As diferenças encontradas entre as amostras tratadas e seus respectivos controles apresentaram significado estatístico. Esses resultados sugerem que a microbiota do solo utilizou Glifosato como fonte de carbono para o seu metabolismo, proporcionando aumento na respiração microbiana. A maior produção de $\mathrm{CO}_{2}$ apresentada por solos com aplicação imediata de Glifosato está relacionada com a degradação do herbicida pelos microrganismos (WARDLE e PARKINSON, 1990).

Os resultados mostraram ainda que ocorreu aumento no desprendimento de $\mathrm{CO}_{2}$ a partir do oitavo dia de incubação, o qual prosseguiu até os 32 dias. Tais resultados diferem dos encontrados por SPRANKLE et. al. (1975) que observaram aumento acentuado na biodegradação de Glifosato na primeira semana de incubação, seguida pela sua diminuição. Verificaram que a adsorção do composto às partículas do solo dificultava a utilização posterior do herbicida pelos microrganismos. De acordo com NOMURA e HILTON (1977) e SOUZA (1994) os microrganismos utilizam a fração que está prontamente biodisponível e que, devido à forte adsorção do Glifosato, a sua degradação é mais acentuada logo após a aplicação no solo.

A comparação entre os solos, com e sem histórico de aplicação prévia de Glifosato, revelou que as amostras ArV6 e LV11 (com histórico de aplicação) apresentaram produção de $\mathrm{CO}_{2}$ em torno de $10 \%$ a $15 \%$ superiores às observadas para as amostras ArV0 e LV0 (sem histórico), respectivamente. $\mathrm{O}$ aumento na liberação de $\mathrm{CO}_{2}$ em solos com histórico de aplicação mostra que os microrganismos presentes nesses solos estão mais adaptados à presença do composto e apresentam capacidade de biodegradação mais acentuada.

\section{FIGURA 1 - QUANTIDADE DE $\mathrm{CO}_{2}$ ACUMULADO NAS AMOSTRAS DE SOLO ArV6 E ArV0 (a) E DAS AMOSTRAS DE SOLO LV11 E LVO (b), DURANTE O PERÍODO DE INCUBAÇÃO DE 32 DIAS $\left(25^{\circ} \mathrm{C}\right.$, NO ESCURO)*}

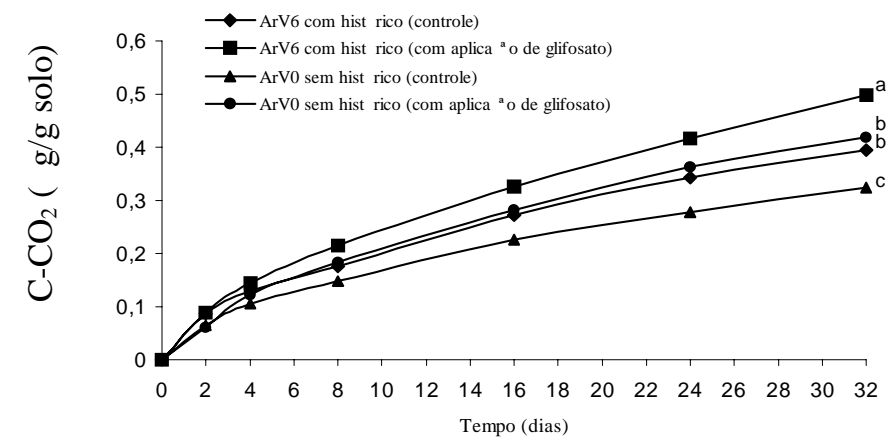

(a)

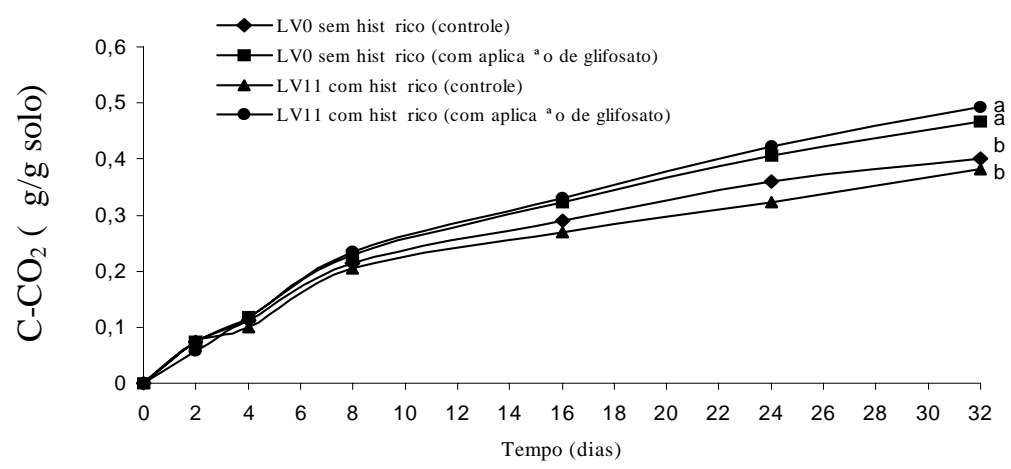

(b)

\footnotetext{
* Média de 3 repetições.
} 
Os resultados obtidos nas análises de resíduos de Glifosato e AMPA das amostras de solos aos $0,8,16$ e 32 dias de incubação são apresentados nas Figuras 2 e 3. Verificou-se diminuição gradativa na quantidade de Glifosato e o aparecimento do metabólito AMPA, que é o primeiro composto formado na degradação microbiana do Glifosato (RUEPPEL et al., 1977). Tais resultados estão de acordo com VEIGA et al. (2001) que avaliaram a dinâmica de Glifosato e AMPA em solo de floresta. Observaram que a concentração do herbicida decresceu enquanto a do seu metabólito aumentou nas primeiras semanas de experimento. Após esse período inicial, a concentração do AMPA decresceu, devido à sua degradação. Neste experimento, a concentração do AMPA provavelmente diminuiria caso fosse monitorada por período superior aos 32 dias, pois a taxa de dissipação do AMPA é menor quando comparada com a do seu precussor Glifosato (NEWTON et al., 1994).

\section{FIGURA 2 - CONCENTRAÇÕES DE GLIFOSATO E AMPA DETECTADAS NOS SOLOS ArV6 (a) E ArVO (a) DURANTE PERÍODO DE INCUBAÇÃO DE 32 DIAS $\left(25^{\circ} \mathrm{C}\right.$, NO ESCURO)*}
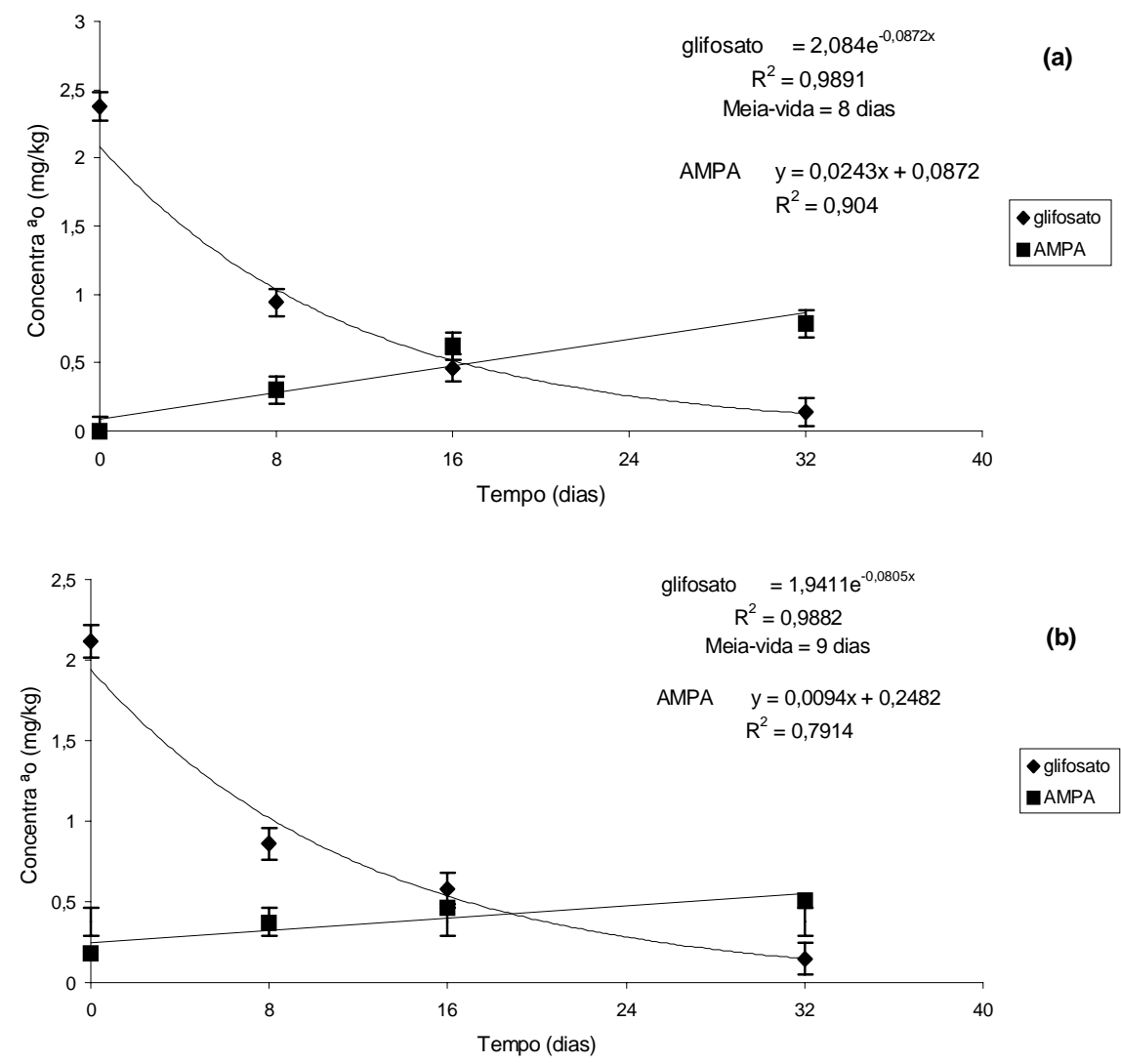

*Média de 3 repetições.

A partir das curvas de degradação apresentadas nas Figuras 2 e 3 foram obtidas as equações que definiram a meia-vida do Glifosato (Figura 2) nos solos avaliados. A meia-vida do Glifosato foi de 8 dias para a amostra de solo ArV6 (com histórico de aplicação) e de 9 dias para a amostra de solo ArV0 (sem histórico de aplicação). Já nas amostras do solo LV (Figura 3), a meia-vida do Glifosato foi de 11 dias para a amostra de solo LV0 (sem histórico de aplicação) e de 22 dias para a amostra de solo LV11 (com histórico de aplicação). A meia-vida menor encontrada nas amostras do solo ArV (Figura 2) pode ter sido conseqüência da maior disponibilidade do Glifosato para biodegradação. Nesses solos, a quantidade de argila encontrada foi menor (Tabela 1), diminuindo conseqüentemente a adsorção do herbicida às partículas de argila. Por outro lado, nas amostras do solo LV que apresentaram maiores quantidades de argila, o Glifosato poderia estar mais adsorvido a essas partículas, impossibilitando maior ataque microbiano e aumentando sua meia-vida nesse solo. Segundo CARLISLE 
e TREVORS (1988), a meia-vida do Glifosato no ambiente pode variar entre pouco menos de uma semana até anos, dependendo do tipo de solo e sua atividade microbiana.

FIGURA 3 - CONCENTRAÇÕES DE GLIFOSATO E AMPA DETECTADAS NOS SOLOS LVO (a) E LV11 (b) DURANTE PERÍODO DE INCUBAÇÃO DE 32 DIAS (25ㄷ, NO ESCURO)*
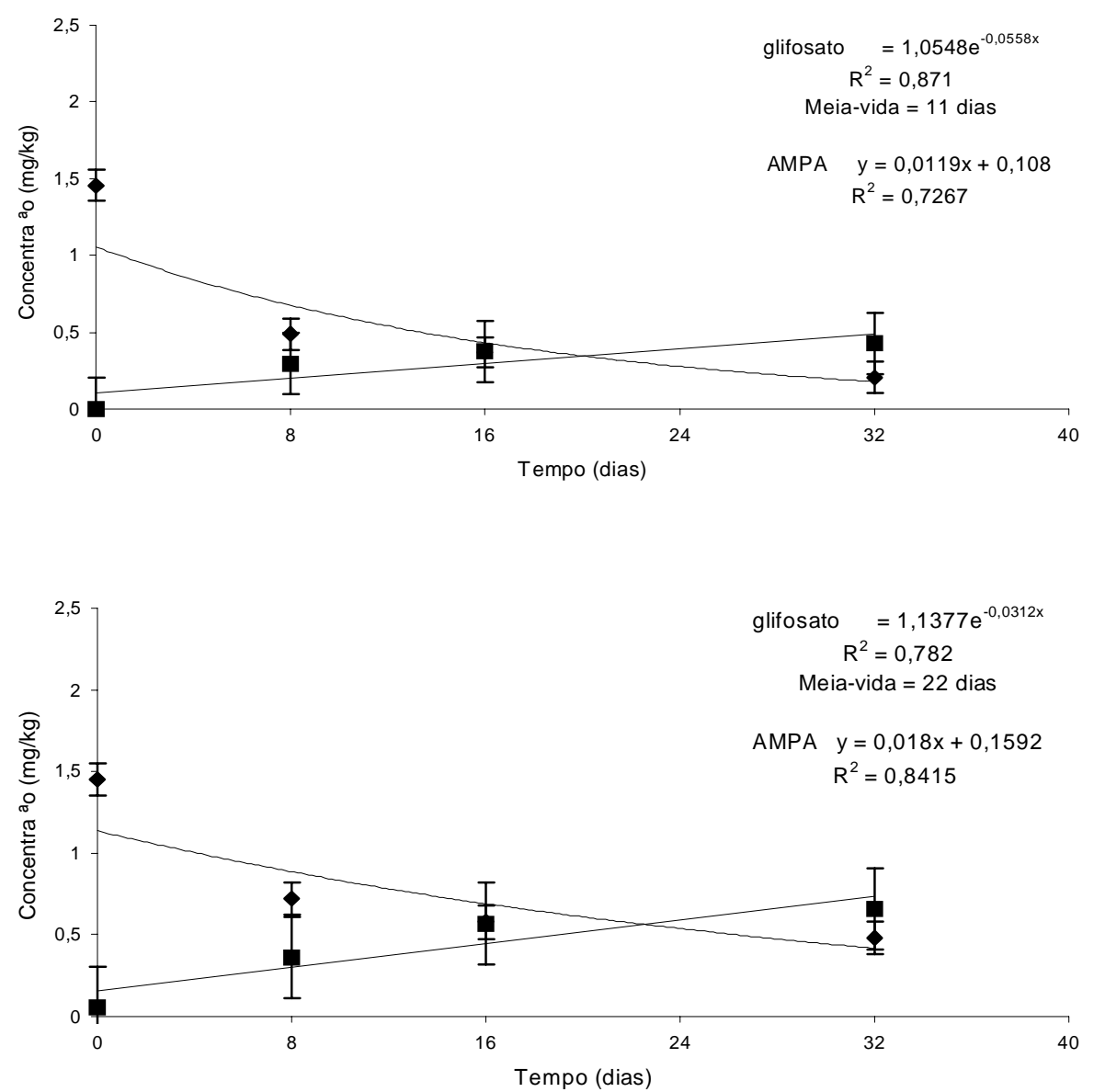

(b)

* Média de 3 repetições.

Na amostra de solo LV0 (sem histórico de aplicação), o Glifosato apresentou meia-vida menor (Figura 3) que na amostra de solo LV11 (com histórico de aplicação). Entretanto, conforme a Figura $1 \mathrm{~b}$, a quantidade de $\mathrm{CO}_{2}$ produzida pela amostra de solo LV11 mostrou-se ligeiramente maior. A quantidade de nutrientes, principalmente o fósforo (VEIGA et al., 2001), disponíveis para os microrganismos na amostra de solo LV0 pode ter estimulado a degradação mais rápida do Glifosato nesse solo, que apresentou meia-vida menor em relação ao solo LV11.

\section{CONCLUSÃO}

A respiração microbiana foi maior nas amostras dos solos que receberam aplicação de Glifosato no início da incubação, mostrando que os microrganismos são os principais responsáveis pela biodegradação do herbicida no solo. A meia-vida do Glifosato foi menor nas amostras de Argissolo do que de Latossolo. 


\begin{abstract}
BIODEGRADATION OF GLYPHOSATE IN TWO BRAZILIAN SOILS

It was evaluated the Glyphosate biodegradation in two Brazilian soil samples, both with and without report of previous herbicide application. Samples of $75 \mathrm{~g}$ of each soil type (3 replicates) were used and Glyphosate was applied in the recommended dose for field condition $\left(2.16 \mathrm{~kg}\right.$ a.i./ha). The assessment of biodegradation was done using the $\mathrm{CO}_{2}$ evolution during a 32 days period. During this period the Glyphosate residues, and its main metabolite were quantified, after extraction and analysis by high performance liquid chromatography. The results showed that Glyphosate was degraded by the soil microorganisms, with formation of its metabolite, the aminomethylphosphonic acid (AMPA). The degradation was faster in the Hapludult than in Hapludox soil.
\end{abstract}

KEY-WORDS: HERBICIDE-BIODEGRADATION; HPLC; GLYPHOSATE; SOILS.

\title{
REFERÊNCIAS
}

1 BARTHA, R.; PRAMER, D. Features of a flask and method for measuring the persistance and biological effects of pesticides in soil. Soil Science, v.100, p.68-70, 1965.

2 CARLISLE, S.M.; TREVORS, J.T. Glyphosate in the environment. Water, Air, Soil Pollution, v.39, p.409-420, 1988.

3 COSTA, M. P. Efeito da matéria orgânica em alguns atributos do solo. Piracicaba, 1983. 137 p. Dissertação (Mestrado) - Escola Superior de Agricultura Luiz de Queiroz, Universidade de São Paulo.

4 COWELL, J.E.; KUNSTMAN, J.L.; NORD, P.J.; STEINMETZ, J.R.; WILSON, G.R. Validation of an analytical residue method for analysis of glyphosate and metabolite: An interlaboratory study. Journal of Agriculture and Food Chemistry, v.34, p.955-960, 1986.

5 EBERBACH, P.L. Applying non-steady-state compartmental analysis to investigate the simultaneous degradation of soluble and sorbed glyphosate (N-phosphonomethyl) glycine in four soils. Pesticide Science, v.52, p.229-240, 1998.

6 FORLANI, G.; MANGIACALLI, A.; NIELSEN, E.; SUARDI, C.M. Degradation of the phosphonate herbicide glyphosate in soil: evidence for a possible involvement of unculturable microorganism. Soil Biology e Biochemistry, v.31, p.991-997, 1999.

7 IBAMA. Instituto Brasileiro de Meio Ambiente e Recursos Renováveis. Manual de testes para avaliação da ecotoxicidade de agentes químicos. Brasília, 1990. 351 p.

8 LIU, C.M.; MCLEAN, P.A.; SOOKDEO, C.C. Degradation of the herbicide glyphosate by members of the family Rhizobiaceae. Applied Environmental Microbiology, v.57, p.1799-1804, 1991.

9 NEWTON, M.; HORNER, L. M.; COWELL, J.E.; WHITE, D.E.; COLE, E.C. Dissipation of glyphosate and aminomethylphosphonic acid in North American forest. Journal of Agriculture and Food Chemistry, v.42, p.1795-1802, 1994.

10 NOMURA, N.S.; HILTON, H.W. The adsorption and degradation of glyphosate in five Hawaiian sugarcane soils. Weed Resource, v.17, p.113-121, 1977.

11 PIMENTEL-GOMES, F.; GARCIA, C.H. Estatística aplicada a experimentos agronômicos e florestais. Piracicaba: FEALQ, 2002. 309 p.

12 ROBERTSON, B.K.; ALEXANDER, M. Growth-linked and cometabolic biodegradation: possible reason for occurrence or absence of accelerated pesticide biodegradation. Pesticide Science, v.41, p.311-318, 1994.

13 RUEPPEL, M. L.; BRIGHTWELL, B. B.; SCHAEFER, J. Metabolism and degradation of glyphosate in soil and water. Journal of Agriculture and Food Chemistry, v.25, p.517-528, 1977.

14 SOUZA, A .P. Atividade de oxyfluorfen, 2,4-D e glifosate, em solos de diferentes texturas na presença e na ausência de composto orgânico. Viçosa, 1994. 71 p. Dissertação (Mestrado) - Universidade Federal de Viçosa.

15 SOUZA, A. P.; FERREIRA, F.A.; SILVA, A. A. Respiração microbiana do solo sob doses de glyphosate e de imazapyr. Planta Daninha, v.17, p.387-398, 1999.

16 SPRANKLE, P.; MEGGITT, W.F. ; PENNER D. Adsorption, mobility, and microbial degradation of glyphosate in the soil. Weed Science, v.23, p.229-234, 1975.

17 VEIGA, F.; ZAPATA, M. L.; FERNANDEZ, M.; ALVAREZ, E. Dynamics of glyphosate and aminomethylphosphonic acid in a forest soil in Galicia, noth-west Spain. The Science of the Total Environment, v.271, p.135-144, 2001.

18 WARDLE, D. A.; PARKINSON, D. Effects of three herbicides on soil microbial biomass and activity. Plant and Soil, V.122, p.21-28, 1990.

\section{AGRADECIMENTOS}

Este trabalho foi financiado pela Fundação de Amparo à Pesquisa do Estado de São Paulo - FAPESP, São Paulo (Processo: 00/06299-8). 